\title{
Microvascular damage - a marker of specific organ involvement in mixed connective tissue disease?
}

\author{
Sylwia Ornowska ${ }^{1 D}$, Marek Chojnowski ${ }^{1}$ ID , Anna Felis-Giemza ${ }^{1}{ }^{\text {ID }}$, tukasz Dudek $^{2}$ ID , \\ Marzena Olesińska ${ }^{1}$ ID
}

${ }^{1}$ Department of Systemic Connective Tissue Diseases, National Institute of Geriatrics, Rheumatology and Rehabilitation, Warsaw, Poland

${ }^{2}$ Medical University of Warsaw, Poland

\begin{abstract}
Mixed connective tissue disease (MCTD) is a complex entity, which incorporates features of systemic lupus erythematosus (SLE), rheumatoid arthritis (RA), systemic sclerosis (SSc) and polymyositis/ dermatomyositis (PM/DM). Nailfold videocapillaroscopy (NVC) is a simple, safe and non-invasive technique of capillary vessel assessment, allowing for qualitative and quantitative assessment of microcirculation. NVC plays a pivotal role in the diagnostic algorithm of connective tissue diseases, especially in systemic sclerosis (SSc). Numerous studies have shown a correlation between organ involvement and disease progression in SSC.

In the current literature, there are limited data on relationship between NVC and organ involvement in MCTD patients. In the present article the relevant literature describing NVC examination in patients with MCTD and comparisons with some clinical situations are discussed.
\end{abstract}

Key words: systemic sclerosis, connective tissue disease, microscopy, microvascular damage.

\section{Introduction}

Mixed connective tissue disease (MCTD) is a complex entity, which incorporates features of systemic lupus erythematosus (SLE), rheumatoid arthritis (RA), systemic sclerosis (SSc) and polymyositis/dermatomyositis (PM/DM) [1]

The clinical presentation and disease severity of MCTD varies significantly among patients, but its most common features are Raynaud's phenomenon (RP), digital edema (puffy fingers), arthralgia, muscle weakness and high titers of anti-U1RNP antibodies. In more severe cases, patients may develop interstitial lung disease (ILD) and, less frequently, pulmonary arterial hypertension $(\mathrm{PAH})$ [2].

Nailfold videocapillaroscopy (NVC) is a simple, safe and non-invasive technique of capillary vessel evaluation, allowing for qualitative and quantitative assessment of microcirculation. NVC plays a pivotal role in diagnostic algorithms of rheumatic connective tissue diseases, especially in differentiating primary and secondary RP, and diagnosing SSc [3].

The extent of microvascular damage observed in SSc, or a so-called scleroderma pattern, has a wellestablished connection with disease progression and internal organ involvement [4]. In other sclerodermaspectrum disorders, such as MCTD and DM, microvascular damage could also be observed and is called a scleroderma-like pattern. However, the association between these alterations and disease severity is still being investigated [3].

\section{Material and methods}

To the authors' knowledge there is little data in the literature on the relationship between changes in NVC and organ involvement in MCTD patients. The relevant literature describing NVC examinations in patients with MCTD was reviewed. 
We retrospectively reviewed our patients' medical history to identify patients with MCTD and different course of the disease. We chose two patients, who both met Kasukawa's criteria for MCTD.

A systematic search of the literature in the electronic database PubMed was conducted using the search terms "mixed connective tissue disease" and "capillaroscopy" in the period from January 2004 to December 2019.

Studies were included if they evaluated capillary alterations in adult patients with MCTD. We were looking for a correlation between capillaroscopic changes and organ involvement in MCTD. Moreover, we used reports describing capillaroscopic changes and their relationship with organ involvement in SSc patients.

From the review of available literature in the last 15 years, finally those directly describing NVC in the context of MCTD were taken into account. Ten original and review articles as well as suggestions of recommendations devoted to this subject were included for analysis and discussion. The reference of the works on this topic to the described clinical pictures (cases) allowed the discussion to be deepened.

\section{Case 1}

The first patient is a 50-year-old man with a 4-year history of MCTD, complicated by ILD and PAH. He was admitted to our department due to exacerbation of RP, pain of multiple peripheral joints, progressive muscle weakness in the shoulder and pelvic girdle, shortness of breath, weight loss and general malaise. He was previously treated with prednisone $5 \mathrm{mg}$ /day, hydroxychloroquine (HCQ) $200 \mathrm{mg} /$ day and sildenafil $60 \mathrm{mg} /$ day.

On admission, the patient's general condition was fair and stable. Physical examination revealed swelling of metacarpophalangeal and proximal interphalangeal joints of both hands, restriction of motion in the shoulder joints and pronounced muscle weakness in the shoulder girdle.

Laboratory findings included elevated erythrocyte sedimentation rate (ESR) and C-reactive protein (CRP), anemia, hypergammaglobulinemia, positive Coombs test, decreased levels of C3 and C4 complement components, and elevated creatine kinase (CK). The anti-nuclear antibody (ANA) titer was extremely high, at $1: 81$ 920, with speckled fluorescence pattern. The ANA profile revealed the presence of anti-U1RNP, anti-Ro, anti-La and anti-Jo1 antibodies.

Chest plain film radiograph and lung high resolution computed tomography (HRCT) showed a reticular interstitial pattern, especially in the lower lung segments. Pulmonary function tests showed restrictive pattern abnormalities and mildly decreased diffusing lung capa- city for carbon monoxide (DLCO). A myogenic pattern was seen in electromyography. In echocardiography, the right systolic ventricular pressure (RSVP) was elevated to $60 \mathrm{~mm} \mathrm{Hg}$. Plain film radiographs of the hands and feet showed soft tissue swelling and erosions in multiple small joints.

In nailfold videocapillaroscopy, a broad spectrum of capillary alterations was observed, ranging from a normal image to complete disorganization of the capillary array typical for active and late scleroderma patterns. It included giant capillaries, numerous ramified capillaries with pathological neoangiogenesis, giant ramified capillaries and severe capillary loss with avascular areas (Figs. 1 and 2).

Due to high disease activity and multi-organ involvement, the dose of glucocorticosteroids was increased to $40 \mathrm{mg} /$ day and the patient was started on intravenous cyclophosphamide $1000 \mathrm{mg} / \mathrm{month}$.

\section{Case 2}

The second patient is a 60-year-old man with a history of MCTD lasting over 20 years. For several months before admission to our department, he experienced systematic increase in the pain and swelling of the wrists and small joints of both hands. Furthermore, he had a history of mild degree ILD, type 2 diabetes, steroid-induced glaucoma and osteoporosis. The current treatment was prednisone $5 \mathrm{mg} /$ day and methotrexate (MTX) $15 \mathrm{mg} /$ week.

On admission, the patient's general condition was good. There were symmetrical swelling and tenderness of the third metacarpophalangeal joints and a slight decrease in strength of the shoulder and pelvic girdle muscles. Laboratory findings were unremarkable with ESR, CRP and CK within normal limits.

High resolution computed tomography of the lungs revealed discrete ground-glass opacities and reticulations, with no progression when compared to previous computed tomography (CT) studies. Pulmonary function tests showed a borderline decrease of DLCO, comparable to previous studies. In echocardiography, RSVP was within normal limits, with no indication of PAH. Plain film radiographs of the hands showed osteoarthritic changes of the small joints.

In nailfold videocapillaroscopy, non-specific alterations were seen, mainly a slight decrease in capillary number to $8-9 / \mathrm{mm}$ and single ramified capillaries. The overall capillary array was normal and no giant capillaries or pathological neoangiogenesis were present (Fig. 3).

Since the disease activity was deemed low and there were no signs of serious internal organ involvement, the 

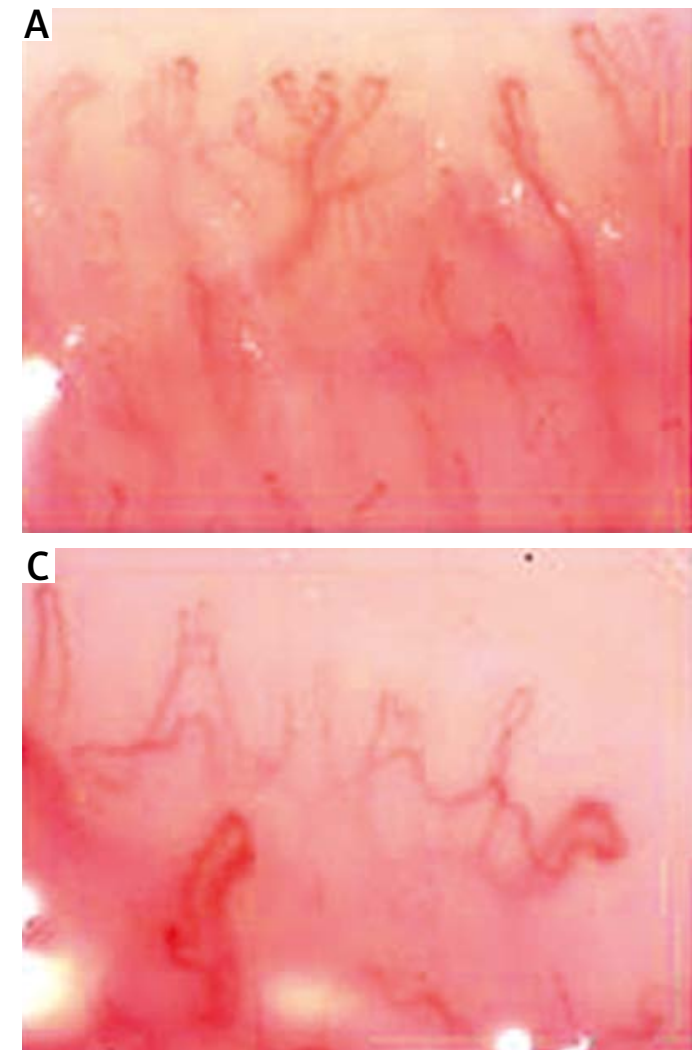

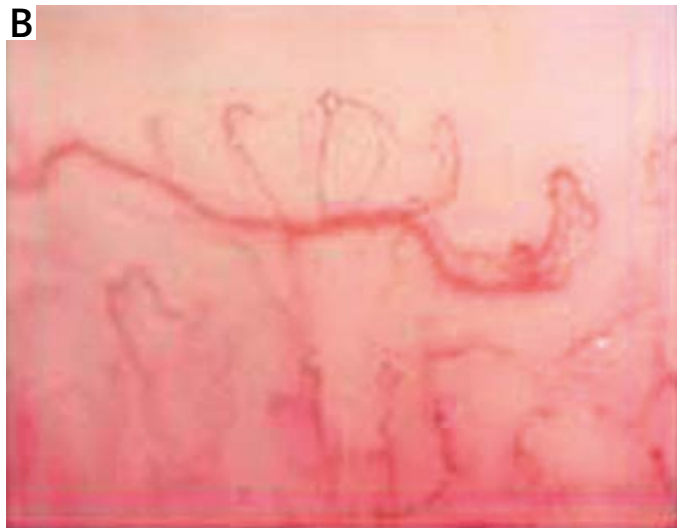

D

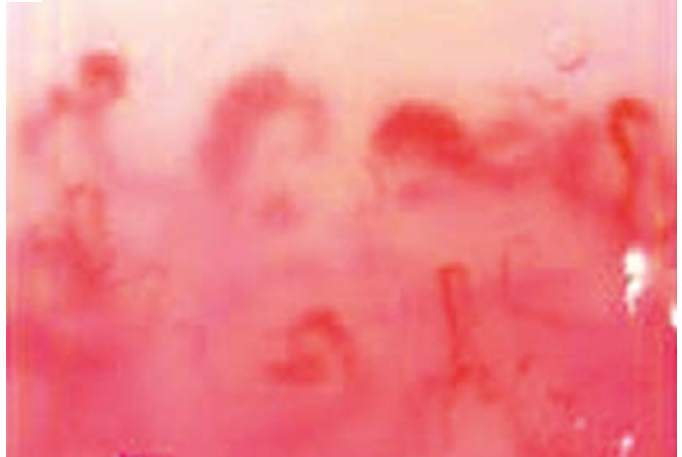

Fig. 1. Full spectrum of scleroderma-pattern capillary alterations: giant capillaries, ramified capillaries with neoangiogenesis and significant capillary loss. Severe clinical course of mixed connective tissue disease, with multi-organ involvement.

A

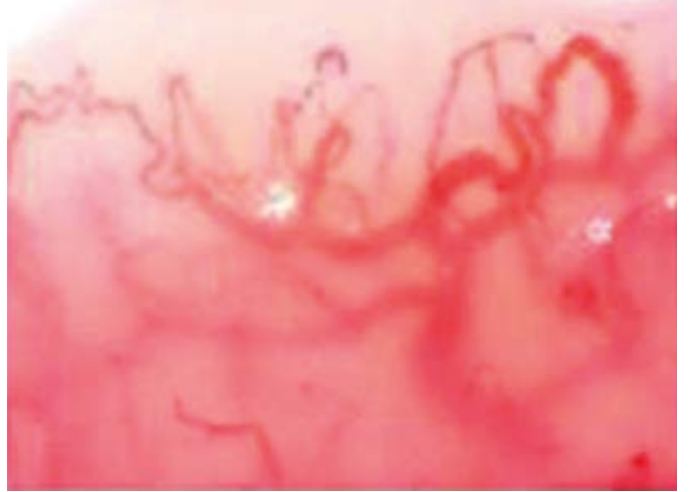

C

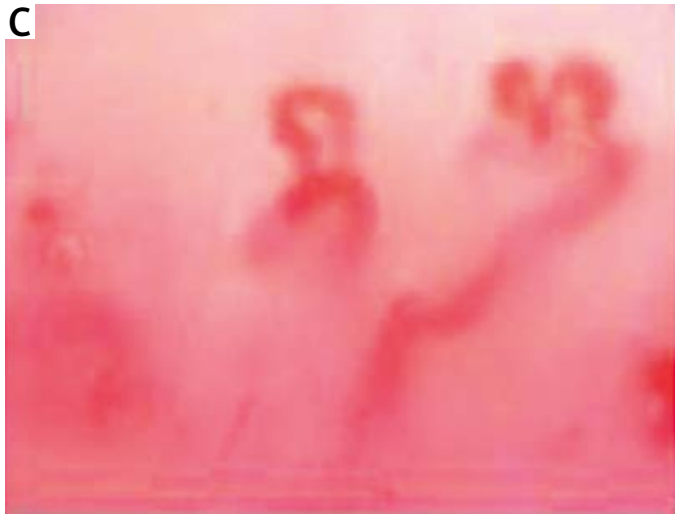

B

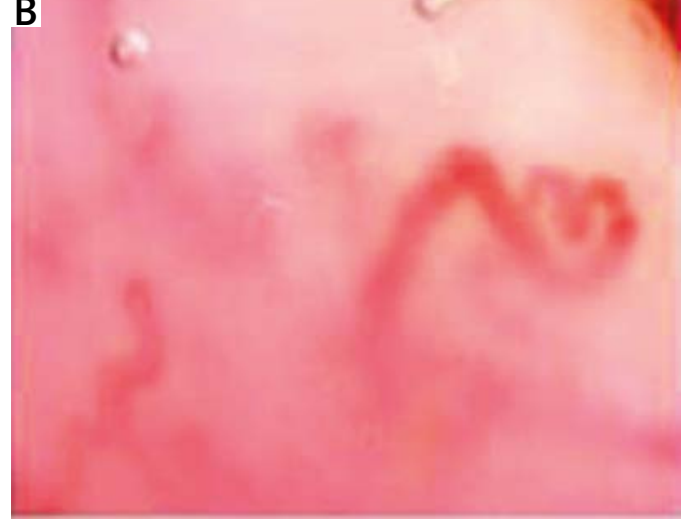

D

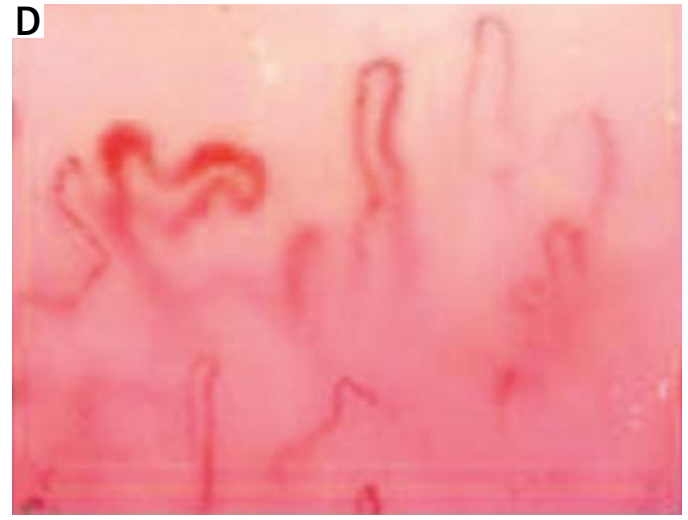

Fig. 2. Giant ramified capillaries. 

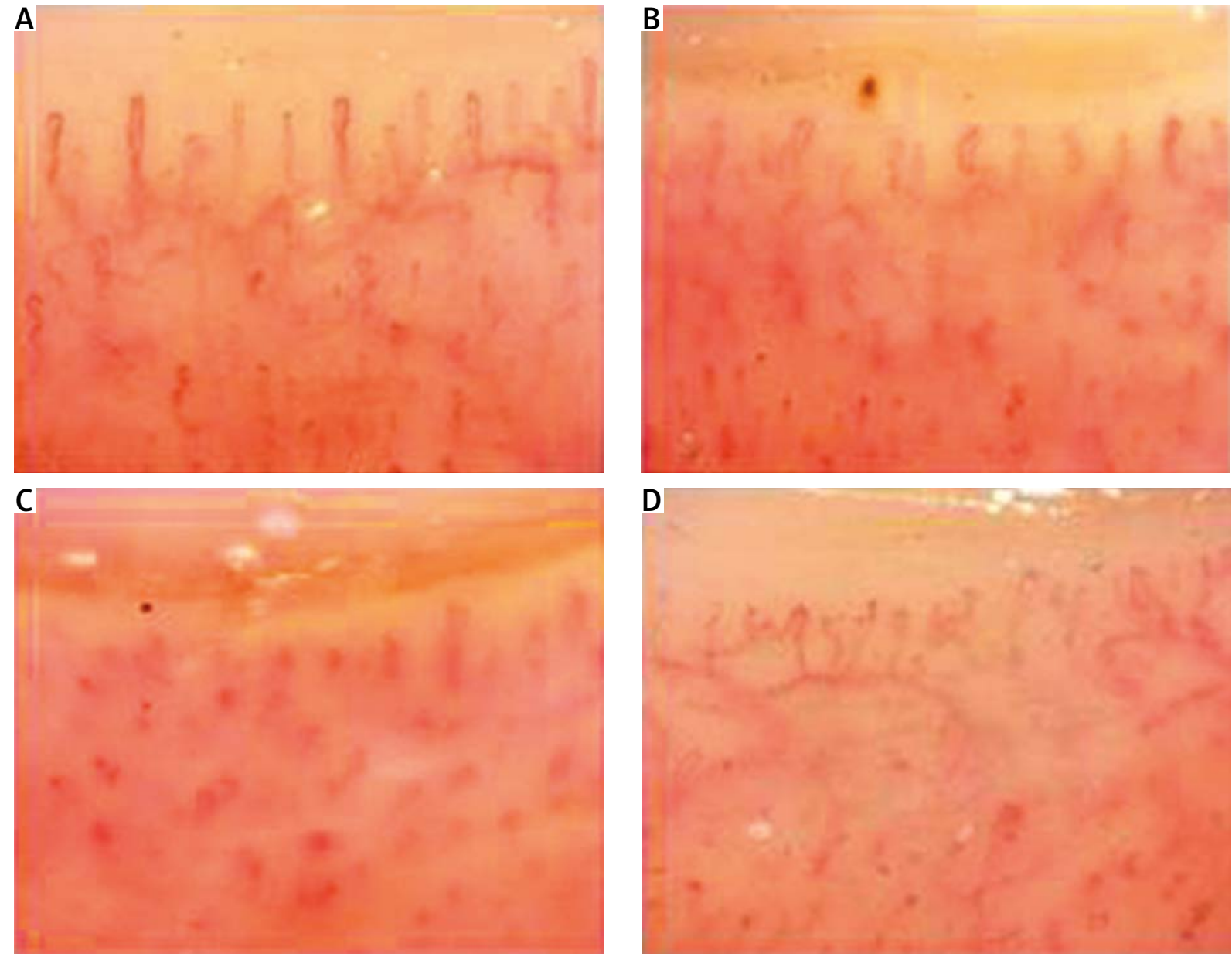

Fig. 3. Non-specific pattern, with slight capillary loss and discrete ramifications. Mild clinical course of mixed connective tissue disease.

Table I. Comparison of disease symptoms in mixed connective tissue disease patients

\begin{tabular}{|lcc|}
\hline Disease symptoms & Case 1 & Case 2 \\
\hline Arthritis & Yes & No \\
\hline Myositis & Yes & No \\
\hline ILD & Yes & Yes \\
\hline PAH & Yes & No \\
\hline Diabetes type 2 & Yes & Yes \\
\hline Atrial fibrillation & Yes & Yes \\
\hline Osteoporosis & No & Yes \\
\hline Raynaud's phenomenon & Yes & Yes \\
\hline
\end{tabular}

Table II. Capillaroscopic pattern in presented cases

\begin{tabular}{|lcc|}
\hline Parameter & Case 1 & Case 2 \\
\hline Number of capillaries & $\begin{array}{c}\text { Severe } \\
\text { capillary loss }\end{array}$ & $\begin{array}{c}\text { Slightly } \\
\text { decrease }\end{array}$ \\
\hline Ramified capillaries & Numerous & Absent \\
\hline Capillary array & Disorganization & Normal \\
\hline Giant capillaries & Present & Absent \\
\hline Neoangiogenesis & Present & Absent \\
\hline Avascular areas & Present & Absent \\
\hline Scleroderma-like pattern & Yes & No \\
\hline
\end{tabular}

MTX dose was adjusted to $25 \mathrm{mg} /$ week, with no additional interventions.

In Tables I and II a comparison between disease symptoms and a comparison of the capillaroscopic picture in the two discussed cases are shown.

\section{Discussion}

In over $80 \%$ of patients with SSC SD pattern can be seen in capillaroscopic examinations [5, 6]. Extent and type of capillary alterations in the nailfold are clearly 
linked with disease severity and the scope of internal organ involvement in patients with SSc. Moreover, the advanced scleroderma patterns (active or late) are associated with increased risk of further disease progression and organ involvement [7-11].

In systemic sclerosis, significant capillary loss is a good indicator of presence and severity of ILD and, to a lesser extent, PAH [12-14]. In mixed connective tissue disease there are only a few reports about a correlation between avascular areas and reduced capillary density in NFC and interstitial lung fibrosis [6, 15-17].

Portuguese authors [18] suggest that capillaroscopy examination performed in patients with undifferentiated connective tissue disease has a prognostic role for the possibility of evolution of the diagnosis.

In more than half of the patients with MCTD, some sort of microvascular damage can be detected. However, especially in comparison with SSc, the correlation of the capillary image with disease progression in MCTD is not clearly established, and the literature on the topic is limited [6].

Wu et al. [19] suggest that NFC pattern has low sensitivity in MCTD. Hajas et al. [10] report that the incidence of a scleroderma-like pattern increases with MCTD duration. Moreover, there are few reports which describe the correlation between the presence of scleroderma-like pattern and the incidence of lung disease or muscle involvement in patients with MCTD [6, 10-12].

Both our patients had ILD and both had a decrease in capillary number. In the first patient, the considerable capillary loss with numerous avascular areas was reflected in severe ILD and coexisting PAH. In the second case, the decrease in capillary number was negligible, and the course of ILD was mild, with little impact on the patient's everyday life.

Giant ramified capillaries are a feature almost uniquely found in DM and anti-synthetase syndrome $[18,19]$, although their relationship with occurrence and degree of muscle involvement is not known [20, 21]. In the first patient, in whom giant ramified capillaries were detected (Fig. 2), evident clinical, biochemical and electromyographical symptoms of active myositis were present. The second patient, with an almost normal capillary image, had no muscle involvement at all.

Pulmonary arterial hypertension and interstitial lung disease are among the leading causes of mortality in mixed connective tissue disease. Khanna et al. [22] recommended that patients with MCTD and other scleroderma-like diseases should be screened for PAH due to high risk of PAH in these patients. Early detection and identification of patients without symptoms is crucial to delaying progression of the disease.

It is clearly visible that now after defining the NVC pattern for systemic sclerosis and assigning this test to the diagnosis of SSc, changes in other systemic scleroses, including MCTD, should be re-analyzed.

\section{Conclusions}

There is an evident connection between NVC and overall disease severity in MCTD. Moreover, the NVC patterns may be helpful in predicting specific organ involvement, e.g. lung disease and myositis.

While these findings have to be confirmed in largegroup, prospective studies, it is reasonable to conclude that NVC should become an integral part of a diagnostic work-up in patients with suspected or established MCTD, since early diagnosis of organ involvement could improve long-term outcomes.

The studies conducted so far and the described clinical observations lead to the conclusion that at present NVC would be more useful for the prognostic analysis of organ involvement and assessment of the prognosis of the course of the disease than for the diagnosis of MCTD.

The authors declare no conflict of interest.

\section{References}

1. Chaigne $B$, Scirè CA, Talarico R, et al. Mixed connective tissue disease: state of the art on clinical practice guidelines. RMD Open 2018; 4 (Suppl 1): e000783, DOI: 10.1136/rmdopen2018-000783.

2. Venables PJ. Mixed connective tissue disease. Lupus 2006; 15 : 132-137, DOI: 10.1191/0961203306lu2283rr.

3. Chojnowski MM, Felis-Giemza A, Olesińska M. Capillaroscopy - a role in modern rheumatology. Reumatologia 2016; 54 : 67-72, DOI: 10.5114/reum.2016.60215.

4. Smith V, Riccieri V, Pizzorni C, et al. Nailfold capillaroscopy for prediction of novel future severe organ involvement in systemic sclerosis. J Rheumatol 2013; 40: 2023-2028, DOI: 10.3899/jrheum.130528.

5. Smith V, Thevissen K, Trombetta AC, et al. Nailfold capillaroscopy and clinical applications in systemic sclerosis. Microcirculation 2016; 23: 364-372, DOI: 10.1111/micc.12281.

6. de Holanda Mafaldo Diógenes A, Bonfá E, Fuller R, Correia Caleiro MT. Capillaroscopy is a dynamic process in mixed connective tissue disease. Lupus 2007; 16: 254-258, DOI: 10.1177/0961203307076517.

7. Ruaro B, Pizzorni C, Paolino S, et al. Correlations between nailfold microvascular damage and skin involvement in systemic sclerosis patients. Microvasc Res 2019; 125: 103874, DOI: 10.1016/j.mvr.2019.04.004.

8. Pizzorni C, Sulli A, Paolino S, et al. Progression of organ involvement in systemic sclerosis patients with persistent "Late" nailfold capillaroscopic pattern of microangiopathy: a prospective study. J Rheumatol 2017; 44: 1941-1942, DOI: 10.3899/jrheum.170485. 
9. Ruaro B, Casabella A, Paolino S, et al. Correlation between bone quality and microvascular damage in systemic sclerosis patients. Rheumatology (Oxford) 2018; 57: 1548-1554, DOl: 10.1093/rheumatology/key130.

10. Hajas A, Szodoray P, Nakken B, et al. Clinical course, prognosis, and causes of death in mixed connective tissue disease. J Rheumatol 2013; 40: 1134-1142, DOI: 10.3899/jrheum.121272.

11. Bernardino V, Rodrigues A, Lladó A, Panarra A. Nailfold capillaroscopy and autoimmune connective tissue diseases in patients from a Portuguese nailfold capillaroscopy clinic. Rheumatol Int 2020; 40: 295-301, DOI: 10.1007/s00296-019-04427-0.

12. Ji LL, Wang H, Zang XH, Zhang ZL. Correlation between nailfold capillaroscopic findings and presence of interstitial lung disease in systemic sclerosis patients. Beijing Da Xue Xue Bao Yi Xue Ban 2018; 50: 501-506 [Article in Chinese].

13. Bredemeier M, Xavier RM, Capobianco K, et al. Nailfold capillaroscopy can suggest pulmonary disease activity in systemic sclerosis. J Rheumatol 2004; 31: 286-294.

14. Corrado A, Correale M, Mansueto N, et al. Nailfold capillaroscopic changes in patients with idiopathic pulmonary arterial hypertension and systemic sclerosis-related pulmonary arterial hypertension. Microvasc Res 2017; 114: 46-51, DOI: 10.1016/j.mvr.2017.06.005

15. Paolino S, Ferrari G, Pizzorni C, et al. Long-term follow-up of nailfold videocapillaroscopic microvascular parameters in mixed connective tissue disease versus systemic sclerosis patients: a retrospective cohort study. Clin Exp Rheumatol 2019; 37 (Suppl 119): 102-107.
16. Lambova, SN, Müller-Ladner U. The role of capillaroscopy in differentiation of primary and secondary Raynaud's phenomenon in rheumatic diseases: a review of the literature and two case reports. Rheumatology Int 2009; 29: 1263-1271, DOI: 10.1007/s00296-009-1019-z.

17. Celińska-Löwenhoff M, Pastuszczak M, Petka, et al. Associations between nailfold capillaroscopy findings and interstitial lung disease in patients with mixed connective tissue disease. Arch Med Sci 2019; 16: 297-301, DOI: 10.5114/ aoms.2018.81129.

18. Cortes S, Clemente-Coelho P. Nailfold capillaroscopy in the evaluation of Raynaud's phenomenon and undifferentiated connective tissue disease. Acta Reumatol Port 2008; 33: 203209 [Article in Portuguese].

19. Wu PC, Huang MN, Kuo YM, et al. Clinical applicability of quantitative nailfold capillaroscopy in differential diagnosis of connective tissue diseases with Raynaud's phenomenon. J Formos Med Assoc 2013; 112: 482-488, DOI: 10.1016/ j.jfma.2012.02.029.

20. Manfredi A, Sebastiani M, Cassone G, et al. Nailfold capillaroscopic changes in dermatomyositis and polymyositis. Clin Rheumatol 2015; 34: 279-284, DOI: 10.1007/s10067-014-2795-8.

21. Chojnowski MM, Nałęcz-Janik J. Regression of microangiopathy in anti-synthetase syndrome. Rheumatology (Oxford) 2017; 56: 724, DOI: 10.1093/rheumatology/kew425.

22. Khanna D, Gladue H, Channick R, et al. Recommendations for screening and detection of connective tissue disease-associated pulmonary arterial hypertension. Arthritis Rheum 2013; 65: 3194-3201, DOI: 10.1002/art.38172. 